\title{
ROOM ON DETERMINANTAL LOCI
}

The Geometry of Determinantal Loci. By T. G. Room. Cambridge, University Press; New York, Macmillan, 1938. $28+483$ pp.

The original purpose of this volume, as stated in the preface, was to provide a systematic account, according to the method and scope of Reye's projective geometry, of the properties of the loci in higher space which are analogous to quadric and cubic surfaces of ordinary space. But these limitations were too narrow; in the final form, synthetic and algebraic methods are used freely, frequently combined in the same problem.

While part of the work is original with the author, and general account of recent contributions, especially of the English School, has been taken, the general scope and procedure of the book are those developed from three sources:

Baker, Principles of Geometry, 6 volumes;

Bertini, Geometria Proiettiva degli Iperspazi;

Segre, Mehrdimensionale Räume (Encyklopädie, III, C7).

Usually citations are not given to original sources, but to the treatment of the problem under consideration in one or another of the three treatises mentioned. Some important recent contributions are not cited at all.

The subject is introduced by concrete examples. Every algebraic plane curve can be defined by a determinant equated to zero. The eliminant of the parameters in two projective pencils of planes defines a quadric surface. The two systems of generators appear at once from its form. Similarly, the equation of a general cubic surface can be so written. Lines on the surface, properties of the double-six, and the plane representation are immediate consequences. Similarly, the space cubic curve is expressed by a two by three matrix of rank one; its systems of bisecants and of tangents are now apparent.

The notation and elaborate symbolism are explained at length. It is a curious fact that almost every locus discussed in the literature on algebraic geometry can be expressed by determinants. Many properties of one locus are shown to be projections or sections of another in a space of a different number of dimensions. Joins, meets, duals (in various spaces) have their usual meanings, and are employed freely, with an appropriate symbolism.

The three fundamental characteristics of any algebraic locus are its dimension, order, and its freedom. The symbol $\left(|p, q|_{r},[n]\right)$ is used to denote the locus of meets of the sets of corresponding linear spaces $[n-p+r]$ of $q$ related stars $] p-1$ [ in $[n]$. The symbol $|p, q|$ means a matrix of $p$ rows, $q$ columns, each element being a linear form in point coordinates in linear space of $n$ dimensions. The number $r$ is the rank of the matrix.

Each such locus can be generated in two ways by systems of projective primes (loci defined by one linear equation in point coordinates), and may have multiple loci of fewer dimensions. This establishes a $(1,1)$ correspondence among the points of one locus, or between the points of two different loci. Thus is opened a new vista of Cremona transformations. However, with few exceptions these are not further developed in the book. It does, on the other hand, use this scheme freely and with skill in developing the representation of a locus upon a linear space.

The plan of the book is to develop a few general theorems and then to elaborate the properties for particular values of $p, q, r, n$, and to show consequences of restricted 
positions of the bases of the generating systems. The effort is made to include all the forms already cited in the literature, and to show relations among results that were derived independently of each other.

The book is hardly suited for systematic reading, but rather as a handbook to assist in marshalling all the pertinent facts concerning a particular problem. In this sense it will frequently be of great value. Among the properties so developed are the pairing theorems and the theorem of the double- $N$. The double-six of lines on a cubic surface furnishes a simple but typical example.

The general manifolds contain relations among the elements of the $p, q$ matrix, and there may be relations among the coefficients of the defining primes. If $n=p q-1$, the elements of the matrix can be taken as the faces of the simplex of reference, and there are no such relations. Such manifolds are called key manifolds. The dual configurations now appear as polar reciprocals as to a quadric primal referred to a selfpolar simplex. In this case the pairing theorems and the double- $N$ take on a more symmetric form.

A chapter on determinantal primals, $p=q, r=p-1$ shows that when $p>3$, the primal is particular. Thus, for $n=3$, a quartic surface requires one condition to be so written. It contains a general sextic curve of genus three. When $p=n$, a determinantal primal can be mapped upon [ $p-1]$.

The last chapter of Part I is concerned with the number of degrees of freedom of projectively generated manifolds. The results are correct for the case of independent elements, but are not always trustworthy for restricted systems. The author carefully points out the limitations of the procedure.

The second part, about 230 pages, is concerned with particular cases; matrices of special forms, symmetric determinants, and so on. The first chapter deals with Veroneseans of quadrics, that is, loci defined by referring the totality of quadrics in $[q]$ to the primes of $q(q+1) / 2$. Particular cases arise when the given system has base elements of various orders and dimensions. The next chapter is concerned with symmetric, partly symmetric, and polar manifolds, Lüroth primals, defined as the sum of reciprocals of linear forms in point coordinates, and the associated Cremona inversion, together with a detailed discussion of Coble's group of reciprocal inversions defined by $n+3$ points in $[n]$. The chapter ends with the treatment of manifolds defining normal elliptic curves of order $n$. Skew-symmetric determinants and Grassmannians of lines are next taken up, including manifolds generated by the duals of lines, which the author calls secunda.

A chapter on normal rational scrolls of $[k]$ 's deals with generalizations of ruled surfaces. Thus, for $k=0$, the curve results. It can be defined by determinants of order 2. The dual is the normal rational developable. For $k=1$, ruled surfaces of lines result; these are given several pages, followed by more general scrolls. A chordal $[s-1]$ is a linear space meeting a scroll in $s$ points. This includes the system of bisecants of a rational normal curve, and more generally defines involutions of order $s$ on the curve.

The $[k]$ 's of $[m]$ can be represented by the points of $[(m-k)(k+1)]$, the base elements being normal rational scrolls. This chapter is a generalization of the representation of lines of [3] by points of [4]. A long chapter on association and incidence theorems is a direct generalization of Segre's famous fifth associated line theorem, which may be formulated as follows: if $a, b, c, d$ are four arbitrary lines of [4], and $a^{\prime}, b^{\prime}, c^{\prime}, d^{\prime}$ are the transversal lines of sets of three of them, then the four [ 3 ]'s $a, a^{\prime}$, and so on have a common line $e$. The five lines $a, b, c, d, e$ form a symmetric set; any plane which meets four of them also meets the fifth. In the same manner the Segre cubic primal in [4] is generalized and various prime representations of it discussed. 
This chapter closes with a summary of the theory of three associated normal rational curves in [4], and its generalizations.

Part III, about 100 pages, is concerned with determinantal primals in [4], and loci contained on it. The first is the Bordiga sextic surface, locus of the meets of corresponding primes of four related sheaves. It contains ten lines, and there are ten planes meeting it in cubic curves which with the lines form a double-ten. The surface is mapped upon a plane. Upon the surface is a system of trisecant lines, one line of which passes through any point of [4]. This at once furnishes a map of the system of trisecants on [3].

Then follow special cases, including those surfaces having a triple point, and others having restricted base points in the representation. A similar surface of order 10 in [8] is generated by [6]'s in four related sheaves. The projection from any four points on it into [4] is the Bordiga sextic. The next chapter considers primals in [4] defined as the vanishing of a general determinant of order 4, the elements of which are arbitrary linear forms in point coordinates. This primal has twenty nodes, and contains four simply infinite systems of lines $h_{i}$, which are discussed in detail. A Bordiga sextic surface on the primal also lies on a cubic primal; the residual intersection is another Bordiga surface. The two systems are distinct and triply infinite.

The primal is mapped upon a [3] by means of its generating systems and the Cremona transformation defined by four bilinear equations among the point coordinates of two [4]'s is discussed. Primes in either space are transformed into general determinantal quartic primals of the other.

The last chapter considers various special cases, including those having a triple point, those containing planes, Veneroni's primal, those defined by a symmetric determinant, polar primals.

Three short appendices are devoted respectively to Veroneseans of primals, the characteristics of various manifolds, and the freedom of manifolds. The volume is provided with an index.

The style is strikingly simple. There are numerous repetitions in description, but these are necessary to prevent ambiguity. At first the reader gets the impression that the symbolism is cumbersome and too much insisted upon, but when he has mastered it, it is at once apparent that descriptions would be much more complicated without it.

The printing and presswork are excellent and the proofreading practically faultless. Two incorrect subscripts are the only cases that might cause confusion.

May this volume, which contains the essence of the entire literature on the subject, be of service compatible with the care and devotion spent in its preparation. 